\title{
Swept-source OCT in patients with multiple evanescent white dot syndrome
}

\author{
Felipe Pereira', Luiz H. Lima ${ }^{1 *}$ D, Alexandre Gomes B. de Azevedo ${ }^{1}$, Claudio Zett ${ }^{1,3}$, Michel E. Farah ${ }^{1,2}$ \\ and Rubens Belfort $\mathrm{Jr}^{1,2}$
}

\begin{abstract}
Background: Swept-source optical coherence tomography (SS-OCT) has a higher scanning rate and longer wavelength in comparison with spectral-domain OCT (SD-OCT), allowing an improved imaging of retinal vascular plexuses and choriocapillaris. The present two patients diagnosed with multiple evanescent white dot syndrome (MEWDS) underwent fundus autofluorescence (FAF), en-face SS-OCT, and SS-OCT angiography (OCTA) imaging, and its features were described and correlated.

Results: The clinical and imaging findings of both cases were consistent with the diagnosis of MEWDS. Color fundus photograph revealed subtle deep retinal white spots in the posterior pole and around the optic disk. FAF showed several hyperautofluorescent lesions corresponding topographically to the subtle deep retinal white lesions observed on color fundus photographs. Cross-sectional SS-OCT showed disruption of the ellipsoid zone (EZ) within the macular area in all study patients. En-face SS-OCT at the level of the outer retina showed lower reflectivity correspondent to the diffuse attenuation due to the EZ disruption on cross-sectional OCT. SS-OCTA demonstrated flow preservation within the retinal vasculature and choriocapillaris.
\end{abstract}

Conclusions: SS-OCT imaging allows a better visualization of the choriocapillaris, and its normal appearance in MEWDS may suggest that the outer retina and photoreceptors represent the primary site of inflammation.

Keywords: Choriocapillaris, MEWDS, Outer retina, Swept-source OCT

\section{Background}

Multiple evanescent white dot syndrome (MEWDS) was first described by Jampol et al. [1] in 1984 as a transient chorioretinal disease of unknown etiology and typically affects young healthy women who present with decreased visual acuity, photopsia, and enlarged blind spot. MEWDS is unilateral in $80 \%$ of the cases, and small white dots in the retinal fundus associated with a granular fovea represent the most characteristic features of this disease. Other less common fundus features include an edematous appearing optic disc and the presence of cells in the vitreous. The majority of MEWDS patients present a spontaneous resolution of all lesions and improvement in visual acuity within weeks $[2,3]$.

Although the precise pathogenesis of MEWDS is unknown, an infectious etiology is thought to be involved

\footnotetext{
*Correspondence: luizlima9@gmail.com

'Department of Ophthalmology, Federal University of São Paulo, Rua

Botucatu, 821, Vila Clementino, São Paulo, SP 04023-062, Brazil

Full list of author information is available at the end of the article
}

in the pathogenesis of the disease since usually a viral prodrome exists [1-3]. The multimodal imaging analysis is very important to study patients with MEWDS because its clinical appearance may be indistinct from other white-dot syndromes. The imaging hallmarks of the disease include punctate hyperfluorescence in a wreath-like pattern of dots and optic disc leakage on fluorescein angiography (FA), widespread nummular hypofluorescent dots on indocyanine green angiography (ICGA), hyperautofluorescent lesions on fundus autofluorescence (FAF), and disruption of outer retina, specifically in the ellipsoid zone (EZ) on spectral-domain optical coherence tomography (SD-OCT) and en-face OCT [4-6].

First analysis of late hypofluorescent lesions with ICGA imaging in MEWDS led to the conjecture that the disease might be due to some impairment in choriocapillaris flow [7]. However, current reports using SD-OCT have implied the outer retina as the site of initial defects in MEWDS [6, 8]. Because swept-source OCT (SS-OCT) has a higher scanning rate and longer wavelength in 
comparison with SD-OCT, it allows an improved imaging of retinal vascular plexuses and choriocapillaris $[9,10]$. In the present report, two patients diagnosed with MEWDS underwent SS-OCT to study the outer retina and choriocapillaris features.

\section{Case report}

\section{Case \#1}

A 28-year-old woman presented with unilateral blurred visual acuity associated with photopsias. Approximately 2 days before the visual symptoms presentation, the patient reported flu-like symptoms, such as fever and headache. The patient had an unremarkable previous medical and ocular history, and there was no recent contact with animals, including cats. Laboratory and systemic imaging tests revealed a normal complete blood count $(\mathrm{CBC})$ and chest and sinus X-rays. Serologies for syphilis, cytomegalovirus, herpes simplex virus, human immunodeficiency virus, Bartonella, Histoplasma capsulatum, Toxoplasma gondii, Toxocara canis, and Borrelia burgdorferi were negative. On ocular examination, the best-corrected visual acuity (BCVA) was 20/20 in the right eye and 20/25 in the left eye. Biomicroscopy of anterior segment, pupillary reactions, and intraocular pressure were normal in both eyes. Color fundus photograph of left eye revealed subtle deep retinal white spots in the posterior pole and around the optic disk. FA demonstrated wreath-like punctate areas of early hyperfluorescence that corresponded to the deep white retinal lesions. Optic disc staining was observed in the late phase of FA. FAF showed several hyperautofluorescent lesions corresponding topographically to the white lesions observed on color fundus photograph. Cross-sectional OCT (DRI Swept
Source OCT Triton, Topcon, Japan) demonstrated disruption of the EZ at the same topography of the spots seen on both the FAF and FA. Punctate hypereflective lesions and hypereflective dots were observed in the outer nuclear layer (ONL) and choroid, respectively. En-face OCT at the level of outer retina (DRI Swept Source OCT Triton, Topcon, Japan) showed multiple hyporeflective spots corresponding to the disruption of the EZ seen on the cross-sectional OCT. There was absence of flow impairment in both the retinal and choroidal vasculature on OCTA (Fig. 1). At 6 month follow-up, the MEWDS lesions spontaneously disappeared, and the visual acuity returned to 20/20.

\section{Case \#2}

A 33-year-old woman presented with unilateral decreased visual acuity associated with photopsias. Approximately 10 days before the visual symptoms presentation, the patient reported flu-like symptoms, such as fever and headache. The patient had an unremarkable previous medical and ocular history, and there was no recent contact with animals, including cats. Laboratory and systemic imaging tests revealed a normal $\mathrm{CBC}$ and chest and sinus X-rays. Serologies for syphilis, cytomegalovirus, herpes simplex virus, human immunodeficiency virus, Bartonella, Histoplasma capsulatum, Toxoplasma gondii, Toxocara canis, and Borrelia burgdorferi were negative. On ocular examination, the BCVA was 20/200 in the right eye and 20/25 in the left eye. Biomicroscopy of anterior segment, pupillary reactions, and intraocular pressure were normal in both eyes. Color fundus photograph of left eye revealed subtle deep retinal white spots in the posterior pole and midperipheral retina. Similar confluent lesions were also observed around the optic disk. FA demonstrated

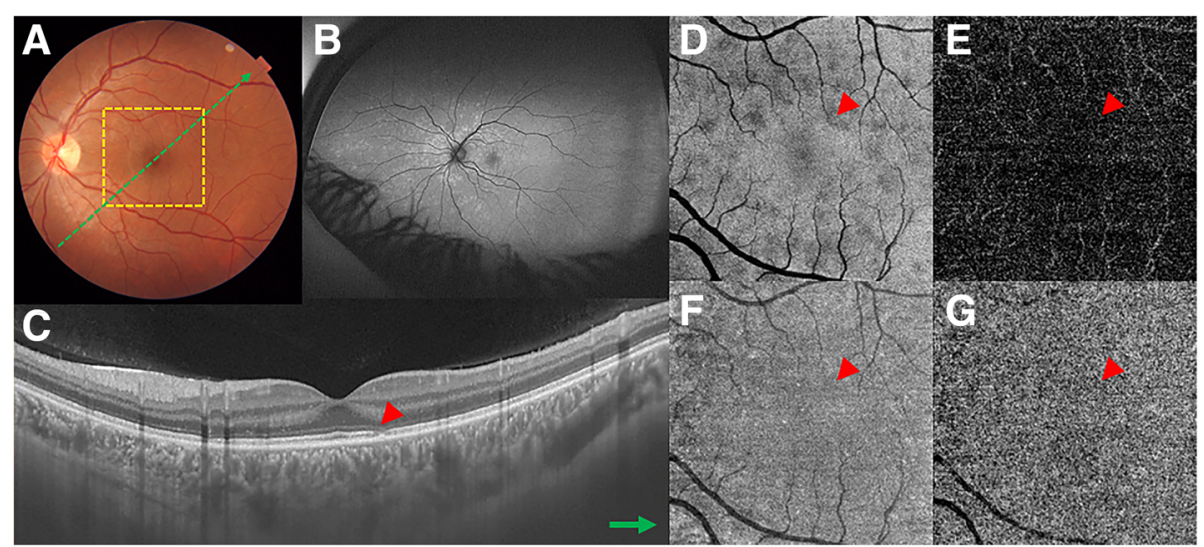

Fig. 1 Case \#1. Multimodal imaging of a 28-year-old woman with multiple evanescent white dot syndrome (MEWDS). a Color fundus imaging shows discrete white lesions in the macular area. $\mathbf{b}$ Wide-field fundus autofluorecence (FAF) depicts a peripapillary hyperautofluorescent area associated with widespread hyperautofluorescent lesions in the retinal fundus. c Swept-source cross-sectional optical coherence tomography $(\mathrm{OCT})$ represented by the green line in image $\mathbf{a}$ shows disruption of the ellipsoid zone (EZ) (red arrowhead). $\mathbf{d}$ En-face OCT at the level of the outer retina shows hyporeflective spots that corresponded to the EZ disruption on cross-sectional OCT (red arrowhead). e- $\mathbf{g}$ OCT angiography (OCTA) of outer retina (e), en-face OCT at the level of choriocapillaris (f), and OCTA of choriocapillaris ( $\mathbf{g}$ ) show homogeneous reflectivity without artifacts or any other abnormalities 
wreath-like punctate areas of early hyperfluorescence that corresponded to the deep white retinal lesions. FAF showed several hyperautofluorescent lesions corresponding topographically to the white lesions observed on color fundus photograph. Cross-sectional OCT (DRI Swept Source OCT Triton, Topcon, Japan) demonstrated punctate hypereflective lesions in the ONL and hypereflective choroidal dots, and disruption of the EZ at the same topography of the spots seen on both the FAF and FA. En-face OCT at the level of outer retina (DRI Swept Source OCT Triton, Topcon, Japan) shows an intrinsic lower reflectivity correspondent to the diffuse attenuation due to the EZ disruption on cross-sectional OCT. There was absence of flow impairment in both the retinal and choroidal vasculature on OCTA (Fig. 2). At 6 month follow-up, the MEWDS lesions spontaneously disappeared, and the visual acuity returned to $20 / 20$.

\section{Discussion}

The diagnosis of MEWDS may be challenging because the lesions are frequently discrete with a characteristic spontaneous resolution [1-3]. The typical MEWDS lesions are observed as deep white spots in the peripapillary and macular areas, according to the natural course of the disease, these lesions disappear and the visual acuity improves to $20 / 20$ during the follow-up period. In the present study cases, the MEWDS lesions were typically hyperautofluorescent and this pattern of FAF may represent increased lipofuscin deposition in the retinal pigment epithelium (RPE) or photoreceptor loss unmasking the natural fluorescence of the underlying RPE [11]. On cross-sectional SD-OCT, disruption of the EZ was observed and corresponded topographically to the hyperautofluorescent spots on FAF. Punctate hypereflective lesions of variable size in the ONL were also observed in our cases. These hypereflective dots in the ONL could be due to lipofuscin migration from the damaged RPE cells into the outer retinal layers or may represent photoreceptor debris shed and accumulated in the outer retina $[6,12]$. Another cross-sectional SD-OCT feature was represented by hypereflective submacular choroidal dots that progressed to the classic presentation of EZ disruption within few days. Fiori et al. noticed that $40 \%$ of their study patients presented hypereflective dots in the inner choroid close to the site of outer retinal layers disruption. These dots are also observed in other choroiditis conditions, and it is possibly due to focal inflammatory cells aggregate [13].

Previous studies using en-face OCT have described two different patterns of lesions in MEWDS patients. Larger hyporeflective and confluent spots colocalized to the level of the EZ and smaller hypereflective dots colocalized to the ONL $[6,14]$. In our cases, the two study patients presented a diffuse reduction of reflectance in the outer retina. This probably happened because the en-face scans were smaller $(4.5 \times 4.5 \mathrm{~mm})$ and there was EZ and interdigitation zone (IZ) discontinuation in this entire area. Possibly, if a larger OCT scan was used, the difference between the affected outer retina (hyporeflective pattern) and healthy outer retina (hypereflective pattern) would be identified. OCTA is a new technology that allows the visualization of chorioretinal vessels flow without dye injection. Although OCTA does not detect vascular leakage, it can identify fine vascular structures that may have important value in cases of choroidal neovascularization associated with MEWDS [15].

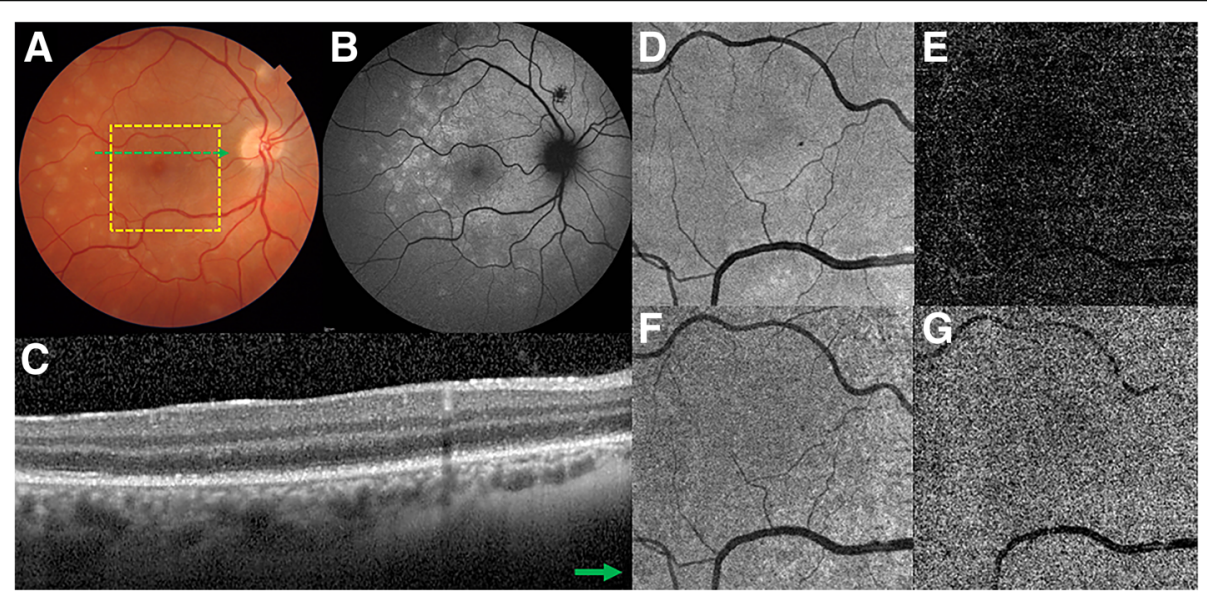

Fig. 2 Case \#2. Multimodal imaging of a 33-year-old woman with MEWDS. a Color fundus imaging shows discrete white lesions in the macular area. b FAF depicts a peripapillary hyperautofluorescent area associated with widespread hyperautofluorescent lesions in the retinal fundus. c Cross-sectional OCT (represented by the green line in image a) shows disruption of the EZ. $\mathbf{d}$ En-face OCT at the level of the outer retina shows an intrinsic lower reflectivity correspondent to the diffuse attenuation due to the EZ disruption on cross-sectional OCT. e-g OCTA of outer retina (e), en-face OCT at the level of choriocapillaris (f), and OCTA of choriocapillaris (g) demonstrate homogeneous reflectivity without artifacts or any other abnormalities 
There is still controversy regarding whether MEWDS is primarily an outer retinal or choriocapillaris/choroidal disease. Jampol et al. implicated photoreceptor damage to underlying RPE dysfunction in their first report of disease [1]. ICGA imaging analysis in MEWDS has associated the hypofluorescent lesions with impairment in choriocapillaris flow [7]. There is evidence of increased choroidal thickness in the acute phase of the disease in both the affected and fellow eyes that returns to the normal choroidal thickness in the convalescent phase of the disease [16]. Fiori et al. observed that not only the choriocapillaris layer was thickened, but also the large choroidal vessels layer when compared with the inactive phase or the fellow eye. The author hypothesized that the initial inflammatory process begins in the inner choroid and then spread to the entire choroid and outer retinal layers [13]. Conversely, several studies using SD-OCT have demonstrated that MEWDS starts in the outer retina and the photoreceptors, specifically in the EZ [17-19]. In their MEWDS series, Pichi et al. [6] demonstrated that the choriocapillaris/choroid and superficial and deep retinal capillary networks were comparable to normal healthy controls. Probably, the hypofluorescence observed on ICGA in MEWDS cases is due to tissue staining rather than choriocapillaris/choroidal injury. Using SS-OCT, Yannuzzi et al. [8] also reported flow preservation within the retinal and choriocapillaris vasculature. Similarly, our SS-OCTA images show that choriocapillaris flow appears to be preserved in the early stages of disease. Therefore, this finding should lead to a more specific research into the pathogenesis of MEWDS. Although oral corticosteroids are sometimes prescribed for MEWDS, our study patients did not take any medication during the disease follow-up as MEWDS is habitually self-resolving and clinical therapy is of undefined aid.

The normal appearance of choriocapillary circulation during the acute phase of disease may suggest that the pathologic process in MEWDS is represented by a primary injury in the outer retina and the photoreceptors. Larger series with long-term follow-up is needed to confirm the preservation of choriocapillaris flow on SS-OCTA in patients with MEWDS.

\section{Abbreviations \\ BCVA: Best-corrected visual acuity; CBC: Complete blood count; EZ: Ellipsoid zone; FA: Fluorescein angiography; FAF: Fundus autofluorescence; ICGA: Indocyanine green angiography; MEWDS: Multiple evanescent white dot syndrome; OCT: Optical coherence tomography; ONL: Outer nuclear layer; RPE: Retinal pigment epithelium; SD-OCT: Spectral-domain optical coherence tomography; SS-OCT: Swept-source OCT}

\section{Availability of data and materials}

We authorize the BMC Ophthalmology journal to reproduce any material described in the manuscript, including new software, databases, and all relevant raw data.

\section{Authors' contributions}

FP participated in conceiving and coordination of the study and helped to draft the manuscript. LL participated in the study coordination and helped to draft the manuscript. AA participated in the study coordination and helped to draft the manuscript. CZ participated in conceiving of the study and helped to draft the manuscript. MF participated in figure production and helped to draft the manuscript. RB participated in the study coordination and helped to draft the manuscript. All authors read and approved the final manuscript.

Ethics approval and consent to participate

Ethics approval and consent to participate were obtained for this case report.

Consent for publication

The consent for publication was obtained from the study patients.

\section{Competing interests}

The authors declare that they have no competing interests.

\section{Publisher's Note}

Springer Nature remains neutral with regard to jurisdictional claims in published maps and institutional affiliations.

\section{Author details}

'Department of Ophthalmology, Federal University of São Paulo, Rua Botucatu, 821, Vila Clementino, São Paulo, SP 04023-062, Brazil. " Vision Institute, São Paulo, Brazil. ${ }^{3}$ Pontificia Universidad Católica de Valparaíso, Valparaíso, Chile.

Received: 21 August 2018 Accepted: 2 October 2018

Published online: 13 October 2018

\section{References}

1. Jampol LM, Sieving PA, Pugh D, Fishman GA, Gilbert H (1984) Multiple evanescent white dot syndrome. I. Clinical findings. Arch Ophthalmol 102: 671-674

2. Tavallali A, Yannuzzi LA (2017) MEWDS, common cold of the retina. J Ophthalmic Vis Res 12:132-134

3. Cahuzac A, Wolff B, Mathis T, Errera MH, Sahel JA, Mauget-Faÿsse M (2017) Multimodal imaging findings in 'hyper-early' stage MEWDS. Br J Ophthalmol 101:1381-1385

4. Gal-Or O, Priel E, Rosenblatt I, Shulman S, Kramer M (2017) Multimodal imaging in an unusual cluster of multiple evanescent white dot syndrome. J Ophthalmol 2017:7535320

5. Dell'omo R, Wong R, Marino M, Konstantopoulou K, Pavesio C (2010) Relationship between different fluorescein and indocyanine green angiography features in multiple evanescent white dot syndrome. $\mathrm{Br} J$ Ophthalmol 94:59-63

6. Pichi F, Srvivastava SK, Chexal S, Lembo A, Lima LH, Neri P et al (2016) En face optical coherence tomography and optical coherence tomography angiography of multiple evanescent white dot syndrome: new insights into pathogenesis. Retina 36:178-188

7. Obana A, Kusumi M, Miki T (1996) Indocyanine green angiographic aspects of multiple evanescent white dot syndrome. Retina 16:97-104

8. Yannuzzi NA, Swaminathan SS, Zheng F, Miller A, Gregori G, Davis JL et al (2017) Swept-source OCT angiography shows sparing of the choriocapillaris in multiple evanescent white dot syndrome. Ophthalmic Surg Lasers Imaging Retina 48:69-74

9. Barteselli G, Bartsch DU, Weinreb RN, Camacho N, Nezgoda JT, Marvasti AH et al (2016) Real-time full-depth visualization of posterior ocular structures: comparison between full-depth imaging spectral domain optical coherence tomography and sweptsource optical coherence tomography. Retina 36: $1153-1161$

10. Huang Y, Zhang Q, Thorell MR, An L, Durbin MK, Laron M et al (2014) Swept-source OCT angiography of the retinal vasculature using intensity differentiation-based optical microangiography algorithms. Ophthalmic Surg Lasers Imaging Retina 45:382-389

11. Freund KB, Mrejen S, Jung J, Yannuzzi LA, Boon CJ (2013) Increased fundus autofluorescence related to outer retinal disruption. JAMA Ophthalmol 131: $1645-1649$ 
12. Hashimoto H, Kishi S (2015) Ultra-wide-field fundus autofluorescence in multiple evanescent white dot syndrome. Am J Ophthalmol 159:698-706

13. Fiore T, laccheri B, Cerquaglia A, Lupidi M, Torroni G, Fruttini D et al (2016) Outer retinal and choroidal evaluation in multiple evanescent white dot syndrome (MEWDS): an enhanced depth imaging optical coherence tomography study. Ocul Immunol Inflamm 11:1-7

14. De bats F, Wolff B, Vasseur V, Affortit A, Kodjikian L, Sahel JA et al (2014) Enface spectral-domain optical coherence tomography findings in multiple evanescent white dot syndrome. J Ophthalmol 2014 2014:928028

15. Nozaki M, Hamada S, Kimura M, Yoshida M, Ogura Y (2016) Value of OCT angiography in the diagnosis of choroidal neovascularization complicating multiple evanescence white dot syndrome. Ophthalmic Surg Lasers Imaging Retina 47:580-584

16. Aoyagi R, Hayashi T, Masai A, Mitooka K, Gekka T, Kozaki K et al (2012) Subfoveal choroidal thickness in multiple evanescent white dot syndrome. Clin Exp Optom 95:212-217

17. Thomas BJ, Albini TA, Flynn HW Jr (2013) Multiple evanescent white dot syndrome: multimodal imaging and correlation with proposed pathophysiology. Ophthalmic Surg Lasers Imaging Retina 44:584-587

18. Marsiglia M, Gallego-Pinazo R, Cunha de Souza E, Munk MR, Yu S, Mrejen S et al (2016) Expanded clinical spectrum of multiple evanescent white dot syndrome with multimodal imaging. Retina 36:64-74

19. Labriola LT, Legarreta AD, Legarreta JE, Nadler Z, Gallagher D, Hammer DX et al (2016) Imaging with multimodal adaptive-optics optical coherence tomography in multiple evanescent white dot syndrome: the structure and functional relationship. Retin Cases Brief Rep 10:302-309

\section{Submit your manuscript to a SpringerOpen ${ }^{\circ}$ journal and benefit from:}

- Convenient online submission

- Rigorous peer review

- Open access: articles freely available online

- High visibility within the field

- Retaining the copyright to your article

Submit your next manuscript at $\boldsymbol{\nabla}$ springeropen.com 